\title{
ON ENDOMORPHISMS OF THE EINSTEIN GYROGROUP IN ARBITRARY DIMENSION
}

\author{
PÉTER E. FRENKEL
}

\begin{abstract}
We determine the automorphisms and the continuous endomorphisms of the Einstein gyrogroup in arbitrary dimension. This generalizes a recent result of L. Molnár and D. Virosztek, who have determined the continuous endomorphisms in the three-dimensional case.
\end{abstract}

\section{The Einstein GYRogroup}

The $n$-dimensional Einstein gyrogroup is the open unit ball $\mathbb{B}^{n}$ in $\mathbb{R}^{n}$, endowed with the binary operation of velocity addition from the special theory of relativity (with the speed of light taken to be 1):

$$
u \oplus v=\frac{1}{1+(u, v)}\left(u+\sqrt{1-|u|^{2}} \cdot v+\frac{(u, v)}{1+\sqrt{1-|u|^{2}}} \cdot u\right) .
$$

Here $(u, v)$ is the inner product of $u$ and $v$, and $|u|=\sqrt{(u, u)}$ is the usual Euclidean norm.

Note that $|u \oplus v|<1$ if $|u|<1$ and $|v|<1$, so $\left(\mathbb{B}^{n}, \oplus\right)$ is an algebraic structure. It satisfies certain axioms that make it a gyrogroup [7]. We shall not need all of the axioms, but let us observe that $u \oplus 0=0 \oplus u=u$ and $u \oplus(-u)=0$ for all $u \in \mathbb{B}^{n}$. The operation $\oplus$ is not associative, but $(-u) \oplus(u \oplus v)=v$ holds for all $u$ and $v$ in $\mathbb{B}^{n}$.

The Einstein gyrogroup is closely related to hyperbolic geometry. If we think of $\mathbb{B}^{n}$ as the Cayley-Klein-Beltrami model of hyperbolic $n$-space, then the map $v \mapsto u \oplus v$ is an isometry of hyperbolic $n$-space for any fixed $u$. When $u \neq 0$, this isometry maps the halfline starting at 0 and passing through $u$ (henceforth referred to as halfline $0 u$ ) onto its sub-halfline starting at $u$.

This implies a well-known fact about commutativity in the Einstein gyrogroup:

Proposition 1. Let $u, v \in \mathbb{B}^{n}$. Then the equality $u \oplus v=v \oplus u$ holds if and only if $u$ and $v$ are linearly dependent (in the usual sense of vector algebra in $\mathbb{R}^{n}$ ).

Proof. If $u$ and $v$ are linearly dependent, then they belong to a diameter of the ball $\mathbb{B}^{n}$. This diameter represents a line $L$ in hyperbolic space. The $L \rightarrow L$ maps $w \mapsto u \oplus w$ and $w \mapsto v \oplus w$ are translations of $L$, so they commute. Hence, $u \oplus v=u \oplus(v \oplus 0)=v \oplus(u \oplus 0)=v \oplus u$ as claimed.

If $u$ and $v$ are linearly independent, then they span a two-dimensional plane, which intersects $\mathbb{B}^{n}$ in a disc. This disc represents a hyperbolic plane.

Research partially supported by ERC Consolidator Grant 648017 and by Hungarian National Foundation for Scientific Research (OTKA), grant no. K109684. 
Hyperbolic isometries preserve angles. Thus, the halfline $u(u \oplus v)$ forms the same angle with the halfline $0 u$ as $0 v$ does. Hence,

$$
\angle 0 u(u \oplus v)=\pi-\angle u 0 v .
$$

Similarly,

$$
\angle 0 v(v \oplus u)=\pi-\angle u 0 v .
$$

If, by way of contradiction, we have $u \oplus v=v \oplus u=w$, then corresponding sides of the triangles $u 0 v$ and $v w u$ have equal length, making the two triangles congruent and implying

$$
\angle u 0 v=\angle v w u .
$$

But then the four angles of the quadrilateral $u 0 v w$ sum to $2 \pi$, which is impossible in the hyperbolic plane.

Corollary 2. The points $x, y, z \in \mathbb{B}^{n}$ are collinear if and only if

$$
((-x) \oplus y) \oplus((-x) \oplus z)=((-x) \oplus z) \oplus((-x) \oplus y) .
$$

Proof. In the Cayley-Klein-Beltrami model, lines of hyperbolic space are represented by chords of the ball $\mathbb{B}^{n}$. Thus, $x, y, z$ are collinear in the ordinary sense of Euclidean geometry if and only if they are collinear as points of hyperbolic space.

The map $w \mapsto(-x) \oplus w$ is an isometry of hyperbolic space, so it preserves collinearity. Thus $x, y$ and $z$ are collinear if and only if $0,(-x) \oplus y$ and $(-x) \oplus z$ are. The claim now follows from Proposition 10

\section{ENDOMORPHISMS AND AUTOMORPHISMS}

An endomorphism of the $n$-dimensional Einstein gyrogroup is a map $f$ : $\mathbb{B}^{n} \rightarrow \mathbb{B}^{n}$ such that

$$
f(u \oplus v)=f(u) \oplus f(v)
$$

for all $u, v \in \mathbb{B}^{n}$. An automorphism is a bijective endomorphism.

Note that any endomorphism $f$ satisfies $f(0)=0$. Indeed, $0 \oplus 0=0$, whence $f(0) \oplus f(0)=f(0)=f(0) \oplus 0$. But $v \mapsto f(0) \oplus v$ is bijective, so $f(0)=0$.

When $n=1$, the Einstein gyrogroup is a group. It is isomorphic to the additive group $(\mathbb{R},+)$ of real numbers. Endomorphisms of this group have been extensively studied, they go under the name of additive functions. Most of them are non-continuous. Moreover, most of the automorphisms of $(\mathbb{R},+)$ are also non-continuous. In fact, the continuous endomorphisms are precisely the linear functions $x \mapsto a x: \mathbb{R} \rightarrow \mathbb{R}$ with fixed $a \in \mathbb{R}$, and there are many further automorphisms, let alone endomorphisms.

Henceforth, we assume $n \geq 2$.

Theorem 3. For $n \geq 2$, automorphisms of the Einstein gyrogroup $\left(\mathbb{B}^{n}, \oplus\right)$ are precisely the restrictions to $\mathbb{B}^{n}$ of the orthogonal transformations of $\mathbb{R}^{n}$.

Proof. Orthogonal transformations of $\mathbb{R}^{n}$ preserve the inner product and therefore the Euclidean norm, so they map $\mathbb{B}^{n}$ bijectively onto itself and satisfy (2) for all $u$ and $v$.

Conversely, if $f$ is an automorphism, then so is its inverse $f^{-1}$. By Corollary 2, both $f$ and $f^{-1}$ map collinear points to collinear points. I.e., $f-$ as 
a self-map of hyperbolic space - maps any line onto a line. In other words, $f$ is a collineation of hyperbolic space. By a well-known result sometimes referred to as the fundamental theorem of hyperbolic geometry $[1,6,6$, $\underline{6}$, any collineation is an isometry for $n \geq 2$. So $f$ is an isometry. It is well known that in the Cayley-Klein-Beltrami model, any isometry of hyperbolic $n$-space fixing 0 is represented by the restriction of an orthogonal transformation.

We now turn to endomorphisms. We urge the reader to solve

Problem 4. For $n \geq 2$, is every endomorphism of the Einstein gyrogroup continuous?

Meanwhile, we wish to classify continuous endomorphisms. For $n=3$, which is the most relevant to physics, this was done by L. Molnár and D. Virosztek [5], while the general case was posed by them as an open problem. Their result relies on a chain of reinterpretations of $\left(\mathbb{B}^{3}, \oplus\right)$. The first step in the chain is an observation of $\mathrm{S}$. $\operatorname{Kim}[2]:\left(\mathbb{B}^{3}, \oplus\right)$ is bicontinuously isomorphic to $(\mathbb{D}, \odot)$, where $\mathbb{D}$ is the set of 2 -square regular density matrices and $A \odot B$ is $\sqrt{A} B \sqrt{A}$ divided by its trace. Molnár and Virosztek show that this in turn is bicontinuously isomorphic to $\left(\mathbb{P}_{2}^{1}, \square\right)$, where $\mathbb{P}_{2}^{1}$ is the set of 2-square positive definite matrices with determinant 1 , and $A \boxminus B=\sqrt{A} B \sqrt{A}$. Then they invoke a result from their previous paper [4, Theorem 1] and deduce from it the classification of the continuous endomorphisms of $\left(\mathbb{P}_{2}^{1}, \square\right)$.

From Theorem 3 of the present paper, using the bicontinuous isomorphisms mentioned above (but in the opposite direction), we infer

Corollary 5. Every automorphism of $(\mathbb{D}, \odot)$ or $\left(\mathbb{P}_{2}^{1}, \square\right)$ is continuous.

Turning to arbitrary dimension, we have

Theorem 6. For $n \geq 2$, continuous endomorphisms of the Einstein gyrogroup $\left(\mathbb{B}^{n}, \oplus\right)$ are precisely the restrictions to $\mathbb{B}^{n}$ of orthogonal transformations of $\mathbb{R}^{n}$ and the map that sends everything to 0 .

For $n=3$, this recovers the classifications of continuous endomorphisms of $\left(\mathbb{B}^{3}, \oplus\right),(\mathbb{D}, \odot)$ and $\left(\mathbb{P}_{2}^{1}, \odot\right)$ given by Molnár and Virosztek in $\underline{\underline{5}}$.

Proof. It is clear that orthogonal transformations and the identically zero map are continuous endomorphisms.

Conversely, let $f$ be an arbitrary continuous endomorphism.

If $f$ is injective, then it is an open map, so its image contains a neighbourhood of 0 . But this neighbourhood generates $\mathbb{B}^{n}$ under $\oplus$, and the image of $f$ is closed under $\oplus$, so $f$ must be surjective, i.e., $f$ is an automorphism. The claim now follows from Theorem 3 .

If $f$ is not injective, then we have a pair $u \neq v$ with $f(u)=f(v)=$ $f(u \oplus((-u) \oplus v))=f(u) \oplus f((-u) \oplus v)$. Let $x=(-u) \oplus v$, then $f(x)=0$ but $x \neq 0$. The diameter $L$ passing through $x$ is a subgroup isomorphic to $(\mathbb{R},+)$. We may choose an isomorphism such that $x$ corresponds to 1 . It is easy to see that $f(y)=0$ for every point of the diameter $L$ that corresponds to a rational number. But then, by continuity, $f(y)=0$ for all $y$ on the diameter $L$. Thus, $f$ is constant on sets of the form $a \oplus L$ and $L \oplus b$. The former sets are lines in hyperbolic $n$-space, i.e., chords of the ball $\mathbb{B}^{n}$. 
The chord $a \oplus L$ passes through $a$ and is parallel to $L$ if $a$ is orthogonal to $L$. The latter sets, when $b \notin L$, are hypercycles in hyperbolic $n$-space, or half-ellipses in $\mathbb{B}^{n}$. The half-ellipse $L \oplus b$ connects the two ends of its major axis $L$ and passes through $b$. It follows that $f$ is constant on any twodimensional open half-disk whose boundary diameter is $L$. By continuity, $f=0$ everywhere.

\section{ACKNOWLEDGEMENTS}

I am grateful to Lajos Molnár and Dániel Virosztek for useful conversations.

\section{REFERENCES}

[1] J. Jeffers, Lost theorems of geometry. Amer. Math. Monthly 107 (2000), no. 9, 800812.

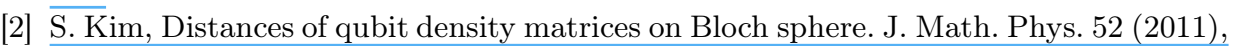
no. 10, 102303, 8 pp.

[3] B. Li and Y. Wang, Transformations and non-degenerate maps, Sci. China Ser. A, Math. 48 (Suppl.) 195-205 (2005).

[4] L. Molnár and D. Virosztek, Continuous Jordan triple endomorphisms of $\mathbb{P}_{2}$, arXiv: $1506.06223 \mathrm{v} 1$

[5] L. Molnár and D. Virosztek, On algebraic endomorphisms of the Einstein gyrogroup, Journal of Mathematical Physics 56 (2015), 082302

[6] E. M. Schröder, Vorlesungen über Geometrie. Band 3, Bibliographisches Inst., Mannheim, 1992.

[7] A.A. Ungar, Analytic hyperbolic geometry and Albert Einstein's special theory of relativity. World Scientific Publishing Co. Pte. Ltd., Hackensack, NJ, 2008.

[8] G. Yao, Fundamental theorem of hyperbolic geometry without the injectivity assumption. Math. Nachr. 284 (2011), no. 11-12, 1577-1582.

Eötvös Loránd University, Department of Algebra and Number Theory, H-1117 Budapest, Pázmány Péter sétány 1/C, Hungary, and Rényi Institute of Mathematics, Hungarian Academy of Sciences, 13-15 Reáltanoda utca, H-1053 BUDAPEST

E-mail address: frenkel.peter@renyi.mta.hu 